\title{
EDD CLUSTERING ALGORITHM FOR WIRELESS SENSOR NETWORKS
}

\author{
Awatef Ben Fradj Guiloufi, Salim El Khediri, Nejah Nasri \\ and Abdennaceur Kachouri \\ National Engineering School of Sfax, Sfax, Tunisia \\ benfradj_awatef@yahoo.fr
}

\begin{abstract}
Power consumption is an important metric tool in the context of the wireless sensor networks (WSNs). In this paper, we described a new Energy-Degree (EDD) Clustering Algorithm for the WSNs. A node with higher residual energy and higher degree is more likely elected as a clusterhead $(\mathrm{CH})$. The intercluster and intracluster communications are realized on one hop. The principal goal of our algorithm is to optimize the energy power and energy load among all nodes. By comparing EDD clustering algorithm with LEACH algorithm, simulation results have showen its effectiveness in saving energy.
\end{abstract}

\section{KEYWORDS}

wireless sensor networks (WSNs), clustering, clusterhead, energy, LEACH.

\section{INTRODUCTION}

A WSNs is a set of nodes called sensors which are able to detect a particular information and to send it to the Base Station (BS). The sensors are inter-connected using a wireless radio communication $([1],[2])$.

The WSNs are characterized by an absence of infrastructure a resources constraints, an heterogeneity and a dynamics structure. For that, it is important to design an auto-organized virtual topology $([3],[4])$.

For these virtual topology, several methods were defined in the literature such as clustering algorithm and the dorsal structure [5].

The clustering procedure is to cut the structure in small zones (called cluster) which are managed by a leader called Cluster Head (CH) ([6], [7]).

For clustering method, the WSNs architecture can be presented into three layers: a sensors nodes which are the receivers of the data, the $\mathrm{CHs}$, and the BS [3].

For WSNs, the important metric tool is generally energy consumption (network lifetime) [8]. In fact, the lifetime is a fundamental parameter in the context of availability in the WSNs ([9], [10]).

Natarajan Meghanathan et al. (Eds) : NeTCoM, CSIT, GRAPH-HOC, SPTM - 2014

pp. 97-104, 2014. C CS \& IT-CSCP 2014

DOI : $10.5121 /$ csit.2014.41308 
In this paper, we proposed an energy efficient clustering algorithm called EDD. The objective of this algorithm is to minimize the energy consumption among all nodes. We propose also to compare between the performances of dynamic clustering and static clustering.

The rest of this paper is organized as follows. Section 2 describes the model of the network. Section 3 presents the proposed algorithm. The simulation results are described in the last section.

\section{EDD CLUSTERING ALGORITHM}

In this section, we define a new clustering algorithm called EDD (Energy-Degree) Clustering Algorithm for WSNs of which the goal is the minimization of the energy consumption and the maximization of network lifetime.

\subsection{Energy Consumption Model}

The energy consumption is generally the most important parameter for WSNs evaluation phase. It depends in fact on the nodes' characteristics (nature of data processing, transmitted power, standby mode, ...), and nodes role during the communication [11].

The consumed energy is defined by this equation [12]:

$$
\mathrm{E}_{\chi}=\mathrm{E}_{\mathrm{c} / \text { capture }}+\mathrm{E}_{\mathrm{c} / \text { treatment }}+\mathrm{E}_{\mathrm{c} / \text { communication }}
$$

Where:

- $\mathrm{E}_{\mathrm{c} / \mathrm{capture}}$ is the consumed energy by a sensor during the capture unit activation.

- $\mathrm{E}_{\mathrm{c} / \text { treatment }}$ is the consumed energy by the sensor during the activation of its treatment unit.

- $\mathrm{E}_{\mathrm{c} / \mathrm{communication}}$ is the consumed energy by the sensor during the activation of its communication unit.

The consumed power by the communication unit is generally very high than the consumed energy by the treatment unit and the capture unit. In fact, the transfer of one bit can consume as much as the execution of a thousands instructions [13]. For this, we can neglect the consumed energy of the capture unit, and the treatment unit compared to the energy consumed by the communication unit. In this case, the equation (1) will be thus:

$$
\mathrm{E}_{\chi}=\mathrm{E}_{\mathrm{c} / \text { communication }}
$$

The communication energy is equal to the sum of the emission energy and the reception energy:

$$
\mathrm{E}_{\mathrm{c} / \text { communication }}=\mathrm{E}_{\mathrm{TX}}+\mathrm{E}_{\mathrm{RX}}
$$

Referring to [14], the transmission the energy and the reception energy can be defined as follows:

$$
\begin{gathered}
\mathrm{E}_{\mathrm{TX}}(\mathrm{K}, \mathrm{d})=\mathrm{E}_{\text {elec }} * \mathrm{~K}+\varepsilon_{\mathrm{amp}} * \mathrm{~K} * \mathrm{~d}^{\lambda} . \\
\mathrm{E}_{\mathrm{RX}}(\mathrm{K})=\mathrm{E}_{\text {elec }} * \mathrm{~K} .
\end{gathered}
$$

Where:

- $\mathrm{K}$ : message length (bits).

- D: distance between transmitting node and receiving node (m). 
- $\quad \lambda$ : of way loss exhibitor, $\lambda>=2$.

- Eelec: emission /reception energy, Eelec $=50 \mathrm{~nJ} / \mathrm{bit}$.

- $\quad$ eamp: transmission amplification coefficient, $\varepsilon a m p=100 \mathrm{pJ} / \mathrm{bit} / \mathrm{m} 2$

\subsection{ClusterHeads election procedure}

Step 1: Each sensor transmits a "hello" message to its neighbors, for the discovery of 1-hop neighborhood.

Step 2: Sensors calculate their weights, the weight is defined as follows:

$$
\text { Weight }(\mathrm{u})=\mathrm{E}_{\mathrm{c} / \mathrm{com}}(\mathrm{u})+1 / \mathrm{D}(\mathrm{u})
$$

Step 3: The sensors distribute their weights to their neighbors.

Step 4: The sensor which has the weakest weight is declared as a $\mathrm{CH}$, it puts its state $=$ " $\mathrm{CH}$ " and transmits a message "clusterhead_elected" (containing its ID) to its neighbors.

Step 5: These laters, after receiving this message, declare themselves like "Nm", transmit to the $\mathrm{CH}$ a message "clusterhead_accepted", and note the identity of their $\mathrm{CHs}$ in their databases.

The EDD clustering algotithm can be described by this organigram:

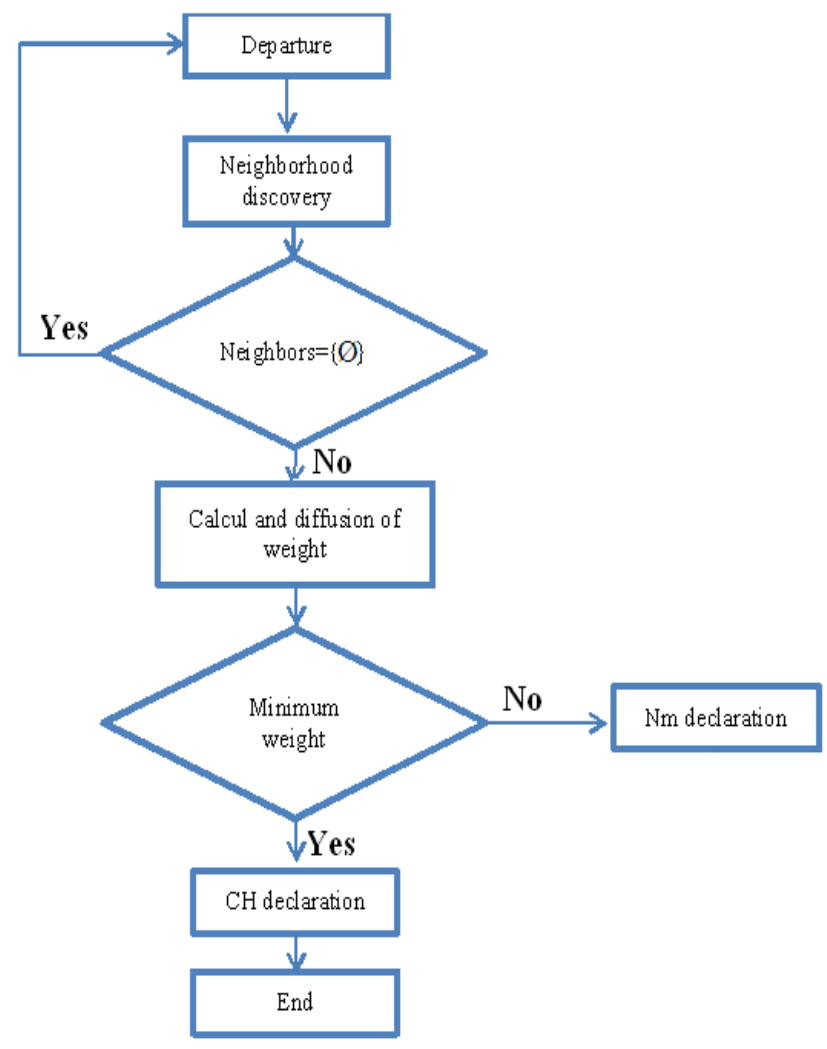

Figure 1. Organigram 


\subsection{Dynamic clustering VS static clustering}

There exist two method of clustering: dynamic clustering and static clustering. For the dynamic clustering, the update of the cluster and $\mathrm{CHs}$ is done each round, in the other hands, the change relates to the totality of the structure.

For the static cluster, the cluster are static and for each cluster, the change only relates to $\mathrm{CH}$, in this case, the number of clusters is always the same and $\mathrm{CH}$ of each cluster changes according to its properties.

\section{SIMULATION RESULTS}

The simulation results are implemented using Matlab 7.0.1 tool. The WSNs is composed of a number of nodes which varies between 10 and 200 nodes.

The simulation of the proposed algorithm was carried out during 10 deactivation intervals $\mathrm{T}$ (standby mode) in a space of $150 \mathrm{~m} \times 150 \mathrm{~m}$ and the range of the nodes (Tx-Arranges) is $40 \mathrm{~m}$. The size of a measured data package for sensors and send towards their clusterheads is 4000 bits. Fig. 2 shows the communication structure of network with 30 nodes.

In Figure. 2, red o represent the $\mathrm{CH}$, yellow triangle represent the ordinary sensor node, blue lines represent the communication between $\mathrm{CH}$ and its members, and black * represent the BS.

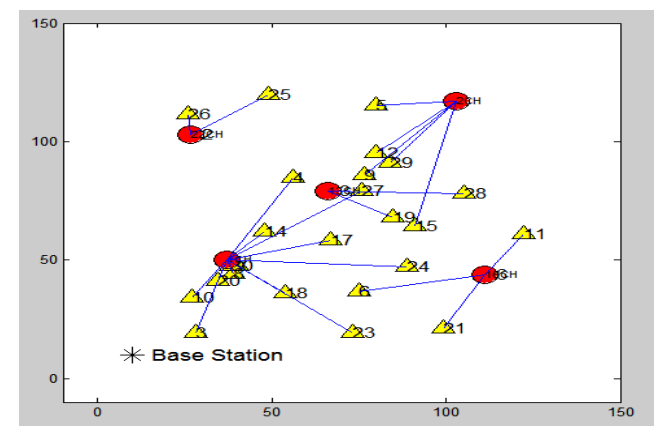

Figure 2. Communication structure of network

Then, we suppose to make a comparison between the dynamic clustering method (DC) and the static clustering method (SC) by applying the proposed clustering algorithm EDD.

Figure. 3 represents the control traffic for both method: EDD- DC indicates that the update is done in a dynamic way and EDD- SC indicates that the method of clustering is static.

We notice that the control traffic is higher for the dynamic method, more control packages are transmitted during the cluster construction phase.

For the static method, control packages are transmitted only if there is a $\mathrm{CH}$ change procedure. A higher control traffic can represent a cause of energy over consumption, but, the energy consumption for the control traffic is generally negligible than that consumed during the transmission of information. 


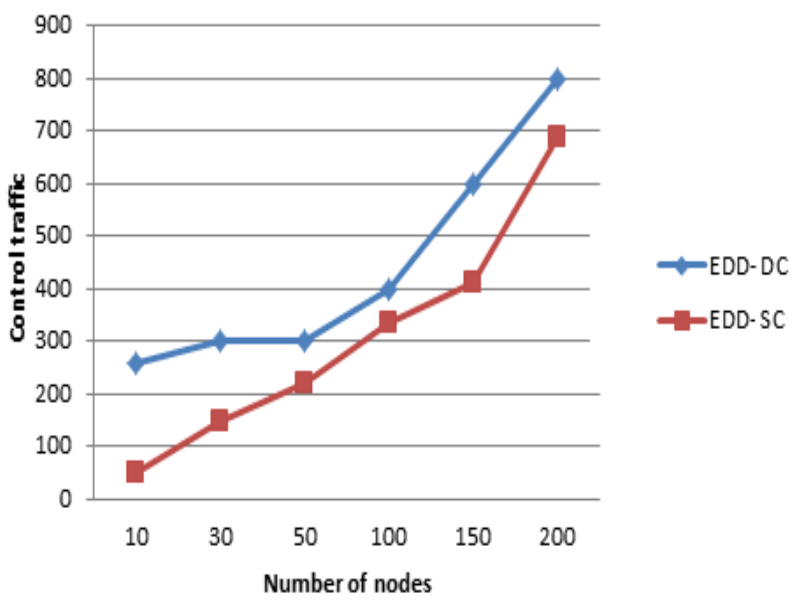

Figure 3. Control traffic

Figure.4 represents the average energy consumption for both methods. We notice that the average energy consumption of the dynamic method is higher, this method consumes more energy during the cluster head election phase and the clusters update phase.

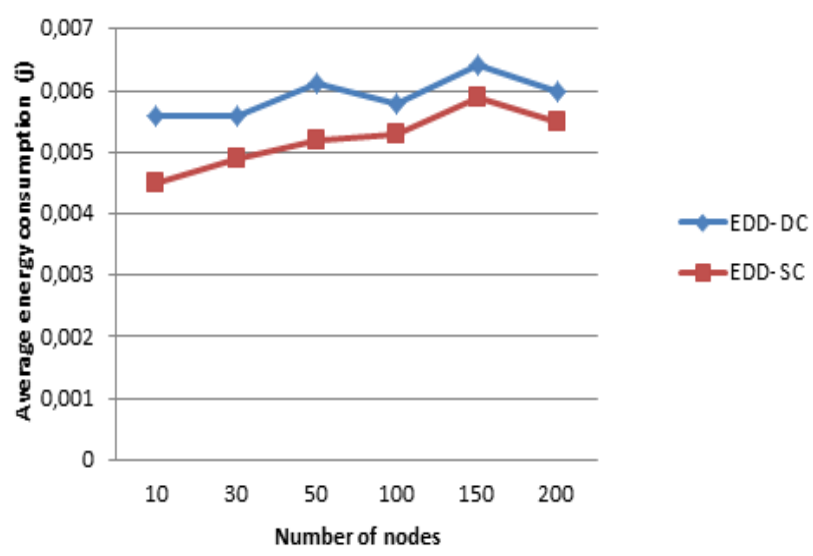

Figure 4. Energy consumption

Figure. 5 represents the first node die for two methods. The best results are given by EDD- DC, this method tends to balance the load of energy consumption between nodes, in this case the network lifetime increases. EDD- SC set up of the static clusters and the update concerne only the $\mathrm{CHs}$. This method can involves the choice of $\mathrm{CHs}$ having a weak energy reserve, which results the crushing of its battery. 


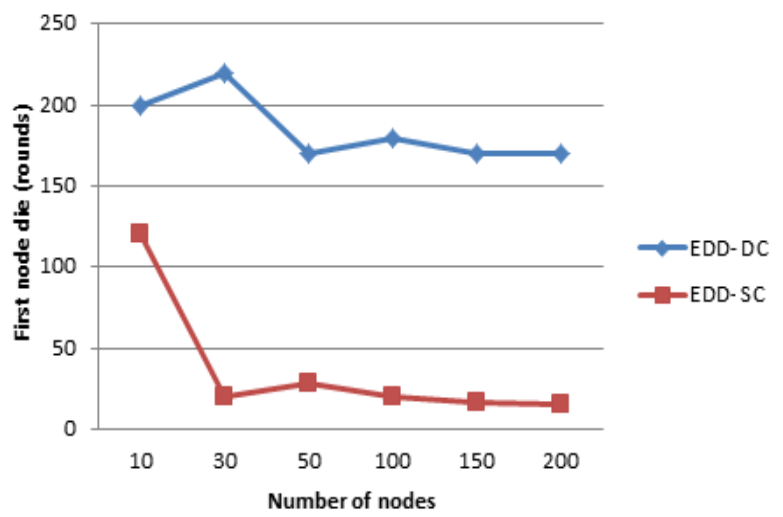

Figure 5. First node die

Among the most known clustering algorithms in literature, we distinguish, LEACH algorithm [8]. LEACH is a famous algorithm which the goal is the minimization of the energy consumption in the WSNs.

We wish in this part to compare our algorithm with the LEACH clustering algorithm. Figure. 6 represents the energy consumption VS the network size. We can note that the values obtained by our algorithm are rather low compared to those obtained by LEACH. These results indicate that our algorithm is more effective and can prolong the network lifetime and ensure its good performance.

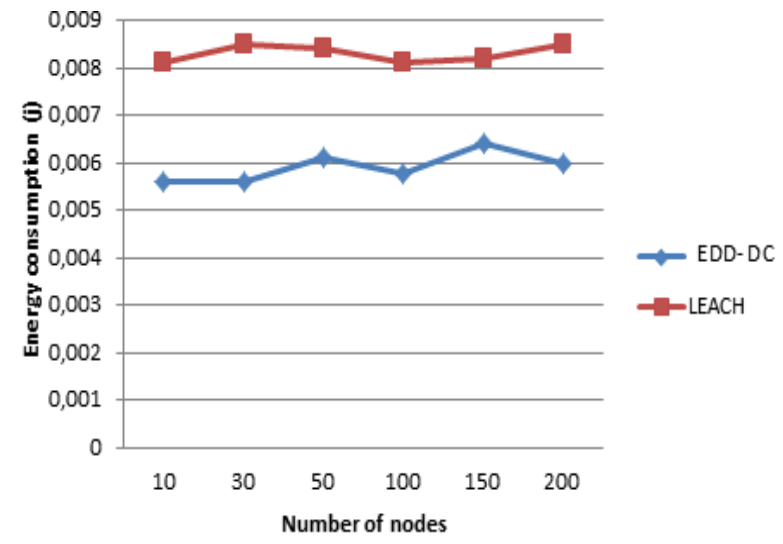

Figure 6. EDD-DC VS LEACH

\section{CONCLUSIONS}

We presented in this paper a new clustering algorithm for the WSNs called EDD. The principal goal of our algorithm is the prolongation of the network life time, two parameters were taken into account for the choice of CHs: the nodes energy consumption and their degree.

The simulation results show that our algorithm is more effective in energy and increase the network lifetime. 


\section{REFERENCES}

[1] C. Tidjane Kone, (2011) 'Conception de l'architecture d'un réseau de capteurs sans fil de grande dimension,' 'PHD, University of Henri Poincaré Nancy I.

[2] G. Chalhoub, (2010) 'Les réseaux de capteurs sans fil', Ph.D, University of Clermont, Auvergne.

[3] L. Samper, (2008) 'Modélisations et analyses de Réseaux de capteurs', PHD, VERIMAG laboratory.

[4] R. Kacimi, (2009)' 'Techniques de conservation d'énergie pour les réseaux de capteurs sans fil,' PHD, TOULOUSE UNIVERSITY.

[5] M. Khan \& J. Misic. (2009) 'On the lifetime of wireless sensor networks," ACM Transactions on Sensor Networks (TOSN), Vol, 5, No. 5.

[6] R. Kuntz, (2010) "Medium access control facing the dynamics of wireless sensor networks, "PHD, University of Strasbourg.

[7] D. Kumar, D. Trilok, C. Aseri \& R. B. Patel, (2009) 'EEHC : energy efficient heterogeneous clustered scheme for wireless sensor networks,' Computer Communication., Vol. 32, No. 4, pp 662667.

[8] W. B. Heinzelman, (2000) 'Application-specific protocol architectures for wireless networks,' PHD, B.S.Cornell University.

[9] Y. Ossama \& S. Fahmy, (2004) 'HEED : A hybrid energy-efficient distributed clustering approach for ad hoc sensor networks,' IEEE Transactions on Mobile Computing, Vol. 3, No. 4, pp $366-379$.

[10]Duan, Changmin \& Hong Fan, (2007) "A distributed energy balance clustering protocol for heterogeneous wireless sensor neworks". In : Proc. Int. Conf. Wireless Communications, Networking and Mobile Computing WiCom. pp. 2469-2473.

[11] V. Raghunathan, C. Schurgers, S. Park \& M.B. Srivastava, (2002) 'Energy- aware wireless microsensor networks,', IEEE Signal Processing Magazine, Vol. 19, No. 2, pp 40 -50.

[12] Mitton, Nathalie, Busson, Anthony \& Fleury, Eric. (2004) 'Self-organization in large scale adhoc networks'. In : Mediterranean ad hoc Networking Workshop (Med-Hoc-Net'04). Bodrum, Turquie.

[13] Wendi Rabiner Heinzelman, Anantha Chandrakasan, \& Hari Balakrishnan, (2000) "Energy-efficient communication protocol for wireless microsensor networks,” In : Proc IEEE 33rd Hawaii International Conference on System Sciences.

[14] G. J. Pottie \& W. J. Kaiser, (2000) 'Wireless integrated network sensors,' Commun. ACM, Vol. 43, No. 5, pp. 51-58.

\section{AUTHORS}

Awatef BENFRADJ was born in Gabes, Tunisia in 1983. She received the engineering degree in Telecommunications and Networks from the University of Gabes, in 2007. In 2009, she obtained the master degree in Networks from Higher School of Communication of Tunisia. Since 2011, she has been a researcher within the laboratory of electronics and information technology, Sfax University.

Salim El Khediri, a Ph.D student at the National School of Engineers of Sfax, Tunisia (Laboratory of Electrical and Information Technology) and in partnership collaboration education with CEDRIC laboratory of CNAM (National Conservatory of Arts and Crafts , Paris). Since 2009 working in the capacity of assistant teacher of computer sciences at the Faculty of Sciences Gafsa - Tunisia.

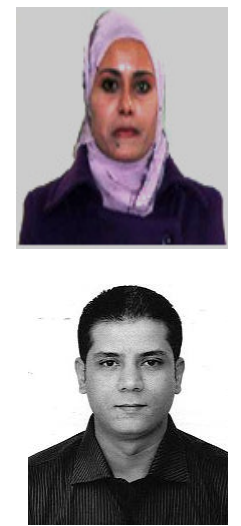


Nejah NASRI was born in Menzel Bouzaienne Village, in 1981. He received the B.S. and M.S. degrees in electronic engineering from the University of Sfax, in 2006 and the $\mathrm{Ph} . \mathrm{D}$. degree in electronic and telecommunication engineering from Toulouse University, France, in 2010. From 2006 to 2009, he was a Research Assistant with Higher Institute of Computer and Communication Techniques (ISITCom), Hammam Sousse, Tunisia. Since 2010, he has been an Assistant Professor with the Informatics Engineering Department, Gafsa University. He is the author of more than 50 articles. His research interests include engineering of wireless sensors networks and wireless underwater communication.

Abdennaceur KACHOURI was born in Sfax, Tunisia, in 1954. He received the engineering diploma from National school of Engineering of Sfax in 1981, a Master degree in Measurement and Instrumentation from National school of Bordeaux (ENSERB) of France in 1981, a Doctorate in Measurement and Instrumentation from ENSERB, in 1983. He "works" on several cooperation with communication research groups in Tunisia and France. Currently, he is Permanent Professor at ENIS School of Engineering and member in the "LETI" Laboratory ENIS Sfax
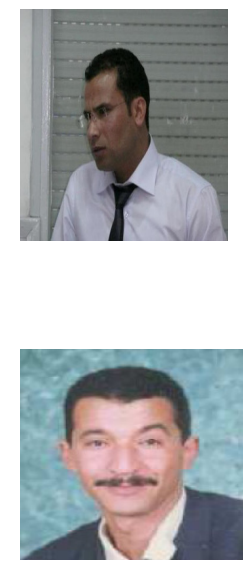